PROCEEDINGS OF THE

AMERICAN MATHEMATICAL SOCIETY

Volume 134, Number 12, December 2006, Pages 3429-3436

S 0002-9939(06)08512-1

Article electronically published on June 9, 2006

\title{
ON THE POLES OF TOPOLOGICAL ZETA FUNCTIONS
}

\author{
ANN LEMAHIEU, DIRK SEGERS, AND WILLEM VEYS
}

(Communicated by Michael Stillman)

\begin{abstract}
We study the topological zeta function $Z_{t o p, f}(s)$ associated to a polynomial $f$ with complex coefficients. This is a rational function in one variable, and we want to determine the numbers that can occur as a pole of some topological zeta function; by definition these poles are negative rational numbers. We deal with this question in any dimension. Denote $\mathcal{P}_{n}:=\left\{s_{0} \mid\right.$ $\exists f \in \mathbb{C}\left[x_{1}, \ldots, x_{n}\right]: Z_{\text {top }, f}(s)$ has a pole in $\left.s_{0}\right\}$. We show that $\{-(n-1) / 2-$ $\left.1 / i \mid i \in \mathbb{Z}_{>1}\right\}$ is a subset of $\mathcal{P}_{n}$; for $n=2$ and $n=3$, the last two authors proved before that these are exactly the poles less than $-(n-1) / 2$. As the main result we prove that each rational number in the interval $[-(n-1) / 2,0)$ is contained in $\mathcal{P}_{n}$.
\end{abstract}

\section{INTRODUCTION}

Denef and Loeser created a new zeta function in 1992, which they called the topological zeta function because of the topological Euler-Poincaré characteristic that turned up in it. Roughly said, the topological zeta function $Z_{t o p, f}$ associated to a polynomial $f$ is a function containing information that we can pick out of each chosen embedded resolution of $f^{-1}\{0\} \subset \mathbb{A}^{n}$. They introduced it in [DL1] in the following way.

Let $f$ be a polynomial in $n$ variables over $\mathbb{C}$ and let $h: X \rightarrow \mathbb{A}^{n}$ be an embedded resolution of $f^{-1}\{0\}$. To define $Z_{t o p, f}$ we need some data related to the embedded resolution $(X, h)$. Let $E_{i}, i \in S$, be the irreducible components of $h^{-1}\left(f^{-1}\{0\}\right)$. Then denote by $N_{i}$ and $\nu_{i}-1$ the multiplicities of $E_{i}$ in the divisor on $X$ of $f \circ h$ and $h^{*}\left(d x_{1} \wedge \ldots \wedge d x_{n}\right)$, respectively. The couples $\left(N_{i}, \nu_{i}\right), i \in S$, are called the numerical data of the resolution $(X, h)$. For $I \subset S$ we also denote $E_{I}:=\bigcap_{i \in I} E_{i}$ and $E_{I}^{\circ}:=E_{I} \backslash\left(\bigcap_{j \notin I} E_{j}\right)$. Further we write $\chi(\cdot)$ for the topological Euler-Poincaré characteristic.

Definition. The local topological zeta function associated to $f$ is the rational function in one complex variable

$$
Z_{t o p, f}(s):=\sum_{I \subset S} \chi\left(E_{I}^{\circ} \cap h^{-1}\{0\}\right) \prod_{i \in I} \frac{1}{N_{i} s+\nu_{i}} .
$$

There is a global version replacing $E_{I}^{\circ} \cap h^{-1}\{0\}$ by $E_{I}^{\circ}$. When we do not specify, we mean the local one.

Received by the editors January 25, 2005 and, in revised form, June 29, 2005.

2000 Mathematics Subject Classification. Primary 14B05, 14J17, 32S05; Secondary 14E15, $32 \mathrm{~S} 25$.

(C)2006 American Mathematical Society 
Denef and Loeser proved that every embedded resolution gives rise to the same function, so the topological zeta function is a well-defined singularity invariant (see DL1]). Once the motivic Igusa zeta function was introduced, they proved this result alternatively in DL2 by showing that this more general zeta function specialises to the topological one.

In particular the poles of the topological zeta function of $f$ are interesting numerical invariants. Various conjectures relate them to the eigenvalues of the local monodromy of $f$; see for example [DL1]. The poles are part of the set $\left\{-\nu_{i} / N_{i} \mid i \in S\right\}$; therefore the $-\nu_{i} / N_{i}$ are called the candidate poles. Note that the poles are negative rational numbers.

A related numerical invariant of $f$ at $0 \in \mathbb{C}^{n}$ is its $\log$ canonical threshold $c_{0}(f)$ which is by definition

$\sup \left\{c \in \mathbb{Q} \mid\right.$ the pair $\left(\mathbb{C}^{n}, c \operatorname{div} f\right)$ is $\log$ canonical in a neighbourhood of 0$\}$.

It is described in terms of the embedded resolution as $c_{0}(f)=\min \left\{\nu_{i} / N_{i} \mid 0 \in\right.$ $\left.h\left(E_{i}\right), i \in S\right\}$ (see [Ko2, Proposition 8.5]). It was studied in various papers of Alexeev, Cheltsov, Ein, de Fernex, Kollár, Kuwata, $\mathrm{M}^{\mathrm{c}}$ Kernan, Mustaţă, Park, Prokhorov, Reid, Shokurov and others. In particular, the sets $\mathcal{T}_{n}:=\left\{c_{0}(f) \mid f \in\right.$ $\left.\mathbb{C}\left[x_{1}, \ldots, x_{n}\right]\right\}$, with $n \in \mathbb{Z}_{>0}$, show up in interesting conjectures; see [Al], Ko1], Ko2], Ku1], Ku2, [McKP], [Pr1], Pr2] and [Sh].

In the context of the topological zeta function, it is natural to similarly study the set

$$
\mathcal{P}_{n}:=\left\{s_{0} \mid \exists f \in \mathbb{C}\left[x_{1}, \ldots, x_{n}\right]: Z_{\text {top }, f}(s) \text { has a pole in } s_{0}\right\} .
$$

The case $n=1$ is trivial: $\mathcal{P}_{1}=\left\{-1 / i \mid i \in \mathbb{Z}_{>0}\right\}$.

From now on we assume that $n \geq 2$. A more or less obvious lower bound for $\mathcal{P}_{n}$ is $-(n-1)$; see [Se1, Section 2.4]. In [SV], the second and the third author studied the 'smallest poles' for $n=2$ and $n=3$. They showed that $\mathcal{P}_{2} \cap\left(-\infty,-\frac{1}{2}\right)=$ $\left\{-\frac{1}{2}-\frac{1}{i} \mid i \in \mathbb{Z}_{>1}\right\}$ and that $\mathcal{P}_{3} \cap(-\infty,-1)=\left\{-1-\frac{1}{i} \mid i \in \mathbb{Z}_{>1}\right\}$. They expected that this could be generalised to

$$
\mathcal{P}_{n} \cap\left(-\infty,-\frac{n-1}{2}\right)=\left\{-\frac{n-1}{2}-\frac{1}{i} \mid i \in \mathbb{Z}_{>1}\right\}, \quad \text { for all } n \in \mathbb{Z}_{>1} .
$$

In particular, they predicted that the lower bound $-(n-1)$ could be sharpened to $-n / 2$. This better bound was recently proven by the second author in $[\mathrm{Se} 2$. In this article we verify for all $n \geq 4$ that $\left\{-(n-1) / 2-1 / i \mid i \in \mathbb{Z}_{>1}\right\} \subset \mathcal{P}_{n}$, and as the main result we show that any rational number in the remaining interval $[-(n-1) / 2,0)$ is a pole of some topological zeta function.

Theorem. For $n \geq 2$ we have $[-(n-1) / 2,0) \cap \mathbb{Q} \subset \mathcal{P}_{n}$.

With the Thom-Sebastiani principle [DL3], $x_{1}^{i}+x_{2}^{2}+\cdots+x_{n}^{2}$ is the obvious candidate to have $-(n-1) / 2-1 / i$ as a pole of its associated topological zeta function. It is not clear a priori that this will be true for all $n$ and $i$. We check this in section 2. For the theorem, however, the key is to find a suitable family of polynomials.

We will put the useful information of the resolution into a diagram, which is called the dual intersection graph. It is obtained as follows. One associates a vertex to each exceptional component in the embedded resolution (represented by a dot) and to each component of the strict transform of $f^{-1}\{0\}$ (represented by a circle). One 
TABLE 1.

\begin{tabular}{|c|c|c|}
\hline$\chi\left(E_{I_{j}}^{\circ}\right)$ & $n$ odd & $n$ even \\
\hline$j=1$ & 1 & -1 \\
$j=2$ & 0 & 1 \\
$j=3$ & 0 & 2 \\
$j=4$ & $n-1$ & $n-2$ \\
\hline
\end{tabular}

also associates to each intersection an edge connecting the corresponding vertices. The fact that $E_{i}$ has numerical data $\left(N_{i}, \nu_{i}\right)$ is denoted by $E_{i}\left(N_{i}, \nu_{i}\right)$.

When the strict transform of $f^{-1}\{0\}$ is irreducible, we will denote it by $E_{0}$. Let $E_{i}$ be an exceptional variety and let $E_{j}, j \in J$, be the components that intersect $E_{i}$ in $X$. We set $\alpha_{j}:=\nu_{j}-\left(\nu_{i} / N_{i}\right) N_{j}$ for $j \in J$; these numbers appear in the calculation of the residue of $Z_{t o p, f}$ in $-\nu_{i} / N_{i}$.

\section{The SET $\left\{-(n-1) / 2-1 / i \mid i \in \mathbb{Z}_{>1}\right\}$ IS A SUbSET OF $\mathcal{P}_{n}$}

Embedded resolution for $x_{1}^{i}+x_{2}^{2}+\cdots+x_{n}^{2}=0, n \geq 4$, with $i$ even. After blowing up $i / 2$ times in the origin, we get an embedded resolution for $f$. We present the dual intersection graph for $i \neq 2$.

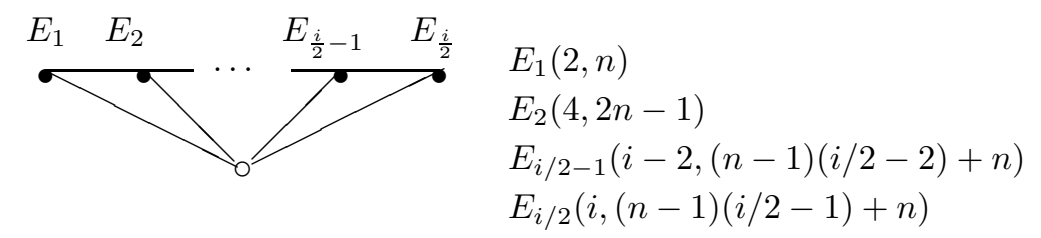

The exceptional variety $E_{i / 2}$ gives the candidate pole $-(n-1) / 2-1 / i$ in which we are interested. If $i \neq 2$, its residue is

$$
\frac{1}{N_{\frac{i}{2}}} \quad\left(\chi\left(E_{I_{1}}^{\circ}\right)+\chi\left(E_{I_{2}}^{\circ}\right) \frac{1}{\alpha_{\frac{i}{2}-1}}+\chi\left(E_{I_{3}}^{\circ}\right) \frac{1}{\alpha_{0}}+\chi\left(E_{I_{4}}^{\circ}\right) \frac{1}{\alpha_{0} \alpha_{\frac{i}{2}-1}}\right),
$$

where $I_{1}=\left\{\frac{i}{2}\right\}, \quad I_{2}=\left\{\frac{i}{2}, \frac{i}{2}-1\right\}, \quad I_{3}=\left\{\frac{i}{2}, 0\right\}, \quad I_{4}=\left\{\frac{i}{2}, \frac{i}{2}-1,0\right\}$. The EulerPoincaré characteristics $\chi\left(E_{I_{j}}^{\circ}\right), 1 \leq j \leq 4$, are put in Table 1 . These are easily computed since $E_{i / 2} \cong \mathbb{P}^{n-1}$, and $E_{i / 2-1}$ and $E_{0}$ intersect $E_{i / 2}$ in a hyperplane and a smooth quadric, respectively. Using that $\alpha_{0}=(3-n) / 2-1 / i$ and $\alpha_{i / 2-1}=2 / i$, some easy calculations yield that the residue is non-zero, for all $n \in \mathbb{N}, n \geq 4$.

When $i=2$, we blow up just once in the origin to get an embedded resolution. By using $\alpha_{0}=\frac{2-n}{2}, \chi\left(E_{I_{1}}^{\circ}\right)=0(n$ even $), \chi\left(E_{I_{1}}^{\circ}\right)=1(n$ odd $)$, we also conclude that here the residue is non-zero.

Embedded resolution for $x_{1}^{i}+x_{2}^{2}+\cdots+x_{n}^{2}=0, n \geq 4$, with $i$ odd. After blowing up $(i+1) / 2$ times in the origin, followed by blowing up once more in $D:=E_{(i+1) / 2} \cap E_{(i-1) / 2} \cong \mathbb{P}^{n-2}$, we get an embedded resolution with the following 
TABLE 2 .

\begin{tabular}{|c|c|c|}
\hline$\chi\left(E_{I_{j}}^{\circ}\right)$ & $n$ odd & $n$ even \\
\hline$j=1$ & 0 & -1 \\
$j=2$ & 0 & 1 \\
$j=3$ & $n-1$ & $n-1$ \\
$j=4$ & 0 & 1 \\
$j=5$ & $n-1$ & $n-2$ \\
\hline
\end{tabular}

dual intersection graph:

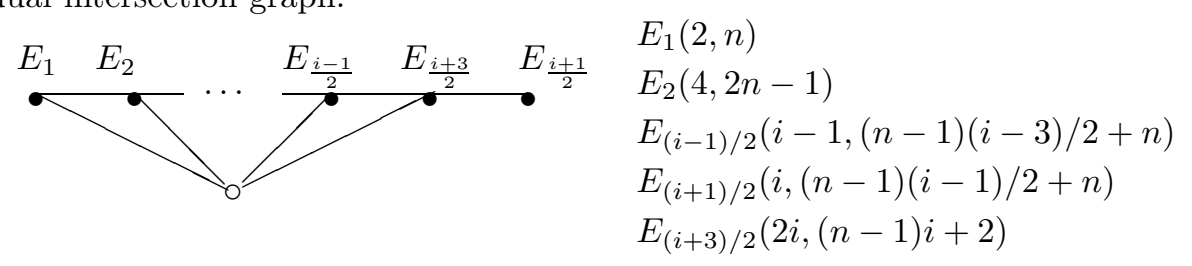

The last exceptional variety has $-(n-1) / 2-1 / i$ as the candidate pole. The relevant subsets in the computation of the residue are $I_{1}=\left\{\frac{i+3}{2}\right\}, \quad I_{2}=\left\{\frac{i+3}{2}, 0\right\}, \quad I_{3}=$ $\left\{\frac{i+3}{2}, \frac{i+1}{2}\right\}, I_{4}=\left\{\frac{i+3}{2}, \frac{i-1}{2}\right\}, I_{5}=\left\{\frac{i+3}{2}, \frac{i-1}{2}, 0\right\}$. Here $E_{(i+3) / 2}$ is a $\mathbb{P}^{1}$-bundle over $D$. For $j=2,3,4$ we have that $E_{I_{j}} \cong D$ and $E_{I_{5}}$ is a smooth quadric. With the Euler-Poincaré characteristics of Table 2 and $\alpha_{0}=(3-n) / 2-1 / i, \alpha_{(i-1) / 2}=1 / i$ and $\alpha_{(i+1) / 2}=(n-1) / 2$, we find that the residue is non-zero, for all $n \geq 4$.

Throwing together these results we obtain

$$
\left\{-\frac{n-1}{2}-\frac{1}{i} \mid i \in \mathbb{Z}_{>1}\right\} \subset \mathcal{P}_{n} .
$$

Now that we checked this expectation, we proceed by proving the theorem.

Remark. Note that $m \in \mathcal{P}_{n-1}$ implies that $m \in \mathcal{P}_{n}$. Indeed, any polynomial $f$ in $n-1$ variables can be considered as a polynomial in $n$ variables. An embedded resolution for $f^{-1}\{0\} \subset \mathbb{C}^{n-1}$ induces the obvious analogous one for $f^{-1}\{0\} \subset$ $\mathbb{C}^{n}=\mathbb{C}^{n-1} \times \mathbb{C}$, and, since $\chi(\mathbb{C})=1$, the two associated topological zeta functions are equal. From this observation it follows that it is sufficient to prove that $[-(n-$ $1) / 2,-(n-2) / 2) \cap \mathbb{Q} \subset \mathcal{P}_{n}$. As we showed in this section that $-(n-1) / 2$ is contained in $\mathcal{P}_{n-1}$ and thus in $\mathcal{P}_{n}$, we restrict ourselves in the next sections to the subset $(-(n-1) / 2,-(n-2) / 2) \cap \mathbb{Q}$.

\section{The $\operatorname{Set}(-1 / 2,0) \cap \mathbb{Q}$ IS A SUbSET OF $\mathcal{P}_{2}$}

Considering what the candidate poles look like in the formula of the topological zeta function written in terms of newton polyhedra (see [DL1]), the number $-(b+2) /(2 a+2 b)$ seems to appear as a candidate pole of the topological zeta function associated to $f(x, y)=x^{a}\left(x^{b}+y^{2}\right)$, where $a$ and $b$ are positive integers. An easy computation yields:

Lemma. When $a$ and $b$ run through $2 \mathbb{Z}_{>0}, a \neq 2$, the quotient $-(b+2) /(2 a+2 b)$ takes all rational values in $(-1 / 2,0)$. 
Taking the lemma into account, the functions $f(x, y)=x^{a}\left(x^{b}+y^{2}\right)$, where $a, b \in 2 \mathbb{Z}_{>0}$ and $a \neq 2$, could be a pretty nice choice to obtain all desired poles. Easy calculations give the following dual resolution graph for $f$ :

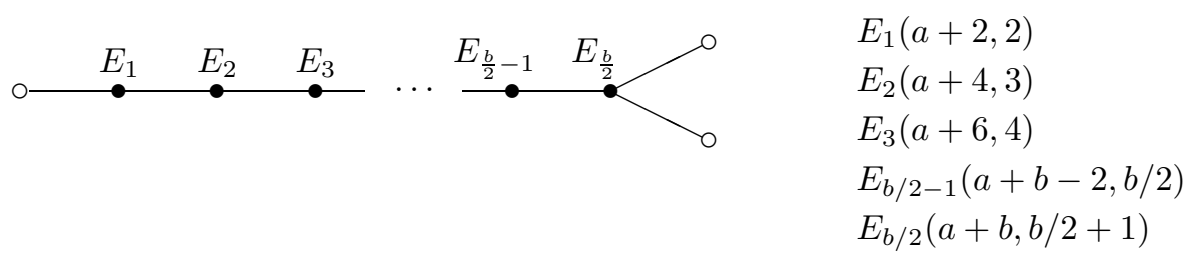

Because $E_{b / 2}$ is intersected three times by other components, Theorem 4.3 in Ve2 allows us to conclude that $-(b+2) /(2 a+2 b)$ is a pole of $Z_{\text {top }, f}$.

4. The $\operatorname{SeT}(-(n-1) / 2,-(n-2) / 2) \cap \mathbb{Q}$ IS A SUbSeT OF $\mathcal{P}_{n}, n \geq 3$

As this set is a translation by $-1 / 2$ of expected poles in dimension $n-1$, the Thom-Sebastiani principle in DL3] is again the motivation why we consider

$$
f\left(x_{1}, \ldots, x_{n}\right)=x_{n}^{2}+\cdots+x_{3}^{2}+x_{1}^{a}\left(x_{1}^{b}+x_{2}^{2}\right),
$$

where $a \in 2 \mathbb{Z}_{>0}$ and $a \neq 2$, to reach the set $(-(n-1) / 2,-(n-2) / 2) \cap \mathbb{Q}$.

Embedded resolution for $z^{2}+x^{a}\left(x^{b}+y^{2}\right)$. Let us first explain in dimension 3 which embedded resolution we choose for $z^{2}+x^{a}\left(x^{b}+y^{2}\right)\left(a, b \in 2 \mathbb{Z}_{>0}, a \neq 2\right)$. We first blow up in the singular locus $\{x=z=0\}$ of $f$ and then always in the singular locus of the strict transform; the first $a / 2$ times this is an affine line and the last $b / 2$ times it is a point. This is the special case for $n=3$ in Table 3 . The dual intersection graph looks as follows:

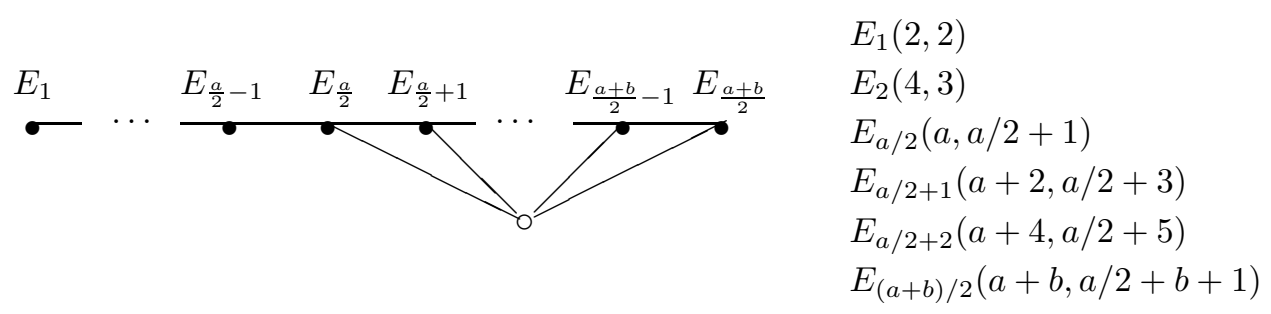

The candidate pole given by the last exceptional surface, $E_{(a+b) / 2}$, is equal to

$$
-\frac{a / 2+b+1}{a+b}=-\frac{b+2}{2 a+2 b}-\frac{1}{2},
$$

and thus covers all rational numbers in $(-1,-1 / 2)$ if $a$ and $b$ run over $2 \mathbb{Z}_{>0}$ and $a \neq 2$.

Embedded resolution for $x_{n}^{2}+\cdots+x_{3}^{2}+x_{1}^{a}\left(x_{1}^{b}+x_{2}^{2}\right), n>3$. The sequence of blow-ups in Table 3 yields an embedded resolution for

$$
f\left(x_{1}, \ldots, x_{n}\right)=x_{n}^{2}+\cdots+x_{3}^{2}+x_{1}^{a}\left(x_{1}^{b}+x_{2}^{2}\right)
$$


TABLE 3 .

\begin{tabular}{|c|c|c|}
\hline $\begin{array}{c}\text { number } i \text { of } \\
\text { blow-up }\end{array}$ & center blow-up & $\begin{array}{c}\text { equation strict transform } \\
\text { in relevant chart }\end{array}$ \\
\hline 1 & $x_{1}=x_{3}=x_{4}=\cdots=x_{n}=0$ & $x_{n}^{2}+\cdots+x_{3}^{2}+x_{1}^{a-2}\left(x_{1}^{b}+x_{2}^{2}\right)$ \\
2 & $x_{1}=x_{3}=x_{4}=\cdots=x_{n}=0$ & $x_{n}^{2}+\cdots+x_{3}^{2}+x_{1}^{a-4}\left(x_{1}^{b}+x_{2}^{2}\right)$ \\
$\vdots$ & $\vdots$ \\
$a / 2$ & $x_{1}=x_{3}=x_{4}=\cdots=x_{n}=0$ & $x_{n}^{2}+\cdots+x_{3}^{2}+x_{1}^{b}+x_{2}^{2}$ \\
$a / 2+1$ & $(0,0, \ldots, 0)$ & $x_{n}^{2}+\cdots+x_{3}^{2}+x_{1}^{b-2}+x_{2}^{2}$ \\
$a / 2+2$ & $(0,0, \ldots, 0)$ & $x_{n}^{2}+\cdots+x_{3}^{2}+x_{1}^{b-4}+x_{2}^{2}$ \\
$\vdots$ & $\vdots$ & $\vdots$ \\
$(a+b) / 2$ & $(0,0, \ldots, 0)$ & $x_{n}^{2}+\cdots+x_{3}^{2}+1+x_{2}^{2}$ \\
\hline
\end{tabular}

based on the previous one for $n=3$. The dual intersection graph here looks as follows:

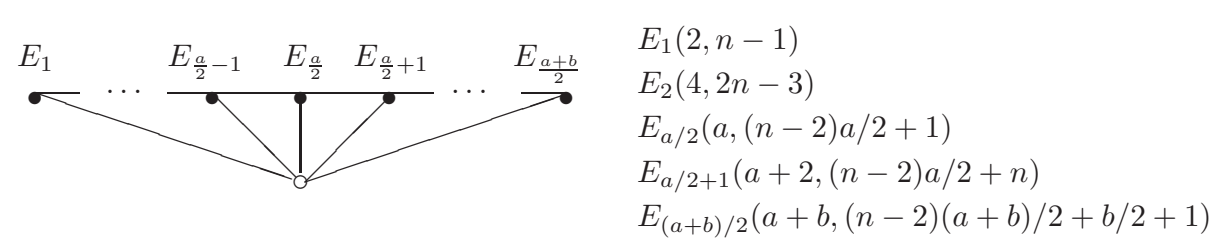

Now $-\nu_{(a+b) / 2} / N_{(a+b) / 2}$ is equal to

$$
-\frac{a / 2+b+1+((a+b) / 2)(n-3)}{a+b}=-\frac{b+2}{2 a+2 b}-\frac{n-2}{2},
$$

which covers the interval $(-(n-1) / 2,-(n-2) / 2) \cap \mathbb{Q}$ when $a$ and $b$ vary in $2 \mathbb{Z}_{>0}$ with $a \neq 2$.

The rational number $-\nu_{(a+b) / 2} / N_{(a+b) / 2}$ is a pole of $Z_{t o p, f}$. For all $n \geq 3$ and $f\left(x_{1}, \ldots, x_{n}\right)=x_{n}^{2}+\cdots+x_{3}^{2}+x_{1}^{a}\left(x_{1}^{b}+x_{2}^{2}\right)$, we calculate the residue of $Z_{t o p, f}$ in $-\nu_{(a+b) / 2} / N_{(a+b) / 2}$. Observe that if $(a+b) /(2+b) \in \mathbb{Z}$, the exceptional variety $E_{(a+b) /(2+b)}$ induces the same candidate pole as $E_{(a+b) / 2}$. The other exceptional varieties always give rise to other candidate poles.

The subsets playing a role in the contribution of $E_{(a+b) /(2+b)}$ to the residue are $J_{1}=\left\{\frac{a+b}{2+b}\right\}, \quad J_{2}=\left\{\frac{a+b}{2+b}, \frac{a+b}{2+b}-1\right\}, J_{3}=\left\{\frac{a+b}{2+b}, \frac{a+b}{2+b}+1\right\}, J_{4}=\left\{\frac{a+b}{2+b}, 0\right\}, \quad J_{5}=$ $\left\{\frac{a+b}{2+b}, \frac{a+b}{2+b}-1,0\right\}, \quad J_{6}=\left\{\frac{a+b}{2+b}, \frac{a+b}{2+b}+1,0\right\}$. Note that when $n=3, E_{(a+b) /(2+b)}$ does not intersect $E_{0}$.

We have that $E_{(a+b) /(2+b)}$ is isomorphic to the cartesian product of $\mathbb{A}^{1}$ and the blowing-up of $\mathbb{P}^{n-2}$ in a point. It is also easy to describe the whole intersection configuration on $E_{(a+b) /(2+b)}$. With the relevant Euler-Poincaré characteristics of Table 4 and $\alpha_{(a+b) /(2+b)-1}=1 / i, \alpha_{(a+b) /(2+b)+1}=-1 / i$, we see that $E_{(a+b) /(2+b)}$ does not give any contribution to the residue in $-\nu_{(a+b) / 2} / N_{(a+b) / 2}$. Alternatively, this is implied by Ve1, Proposition 6.5]. This means we only have to take the contribution of $E_{(a+b) / 2}$ into account. 
TABLE 4.

\begin{tabular}{|c|c|c|}
\hline$\chi\left(E_{J_{k}}^{\circ}\right)$ & $n$ odd & $n$ even \\
\hline$k=1$ & 0 & 0 \\
$k=2$ & 1 & 0 \\
$k=3$ & 1 & 0 \\
$k=4$ & 0 & 0 \\
$k=5$ & $n-3$ & $n-2$ \\
$k=6$ & $n-3$ & $n-2$ \\
& & \\
\hline
\end{tabular}

To compute this contribution the relevant subsets for the summation in the formula of the topological zeta function are $I_{1}=\left\{\frac{a+b}{2}\right\}, I_{2}=\left\{\frac{a+b}{2}, \frac{a+b}{2}-1\right\}, I_{3}=$ $\left\{\frac{a+b}{2}, 0\right\}, I_{4}=\left\{\frac{a+b}{2}, \frac{a+b}{2}-1,0\right\}$. The Euler-Poincaré characteristics $\chi\left(E_{I_{j}}^{\circ}\right), 1 \leq$ $j \leq 4$, are the same as those given in Table 1 , and we have $\alpha_{0}=-((n-4) a+$ $(n-3) b+2) /(2(a+b))$ and $\alpha_{(a+b) / 2-1}=(2-a) /(a+b)$.

As the residue then is equal to

$$
\begin{aligned}
& \frac{(-2+3 a+2 b)(n a-2 a-b+n b+2)}{(-2+a)(a+b)(n a-4 a+2+n b-3 b)} \quad \text { for } n \text { odd and } \\
& \frac{(2+b)(n a-2 a-b+n b+2)}{(-2+a)(a+b)(n a-4 a+2+n b-3 b)} \quad \text { for } n \text { even, }
\end{aligned}
$$

we find that $-\left(\nu_{(a+b) / 2}\right) /\left(N_{(a+b) / 2}\right)=-(b+2) /(2 a+2 b)-(n-2) / 2$ is a pole of $Z_{t o p, f}$.

We conclude that $(-(n-1) / 2,-(n-2) / 2) \cap \mathbb{Q} \subset \mathcal{P}_{n}$, for all $n \geq 3$.

\section{SOME REMARKS}

(1) Instead of achieving this result with the method of resolution of singularities one can find the poles of the topological zeta function of the polynomials

$$
x_{n}^{2}+\cdots+x_{3}^{2}+x_{1}^{a}\left(x_{1}^{b}+x_{2}^{2}\right) \quad \text { and } \quad x_{n}^{2}+\cdots+x_{2}^{2}+x_{1}^{i}
$$

with the help of Newton polyhedra. Indeed, we can write down the topological zeta function for these polynomials using the formula of Denef and Loeser in DL1]. For example if $f\left(x_{1}, \ldots, x_{n}\right)=x_{n}^{2}+\cdots+x_{3}^{2}+x_{1}^{a}\left(x_{1}^{b}+x_{2}^{2}\right)$, where $a$ and $b$ are positive even integers and $a \neq 2$, put $A:=(a+b) s+1+b / 2+(n-2)(a+b) / 2$ and $B:=a s+1+(n-2) a / 2$. We get

$$
\begin{aligned}
Z_{\text {top }, f}(s)= & (n-1) \frac{b}{2 A B}+\frac{1}{A}+(n-2) \frac{a}{2 B} \\
& +\frac{s}{s+1}\left(\sum_{d=1}^{n-1}\left(\begin{array}{c}
n-2 \\
d+1
\end{array}\right)\left(\frac{a}{2 B}+\frac{b}{2 A B}\right)(-2)^{d}\right. \\
& \left.+\sum_{d=1}^{n-1}\left(\begin{array}{c}
n-1 \\
d
\end{array}\right) \frac{1}{A}(-2)^{d}+\sum_{d=1}^{n-2}\left(\begin{array}{c}
n-2 \\
d
\end{array}\right) \frac{b}{2 A B}(-2)^{d}\right) .
\end{aligned}
$$

Handling the problem in this way leads to the same results. One just has to be careful with the dual cones of some faces, namely those that are not a rational simplicial cone. 
(2) With a similar definition of $\mathcal{P}_{n}$ in each case, the same results hold for local and global versions of the motivic zeta function, the Hodge zeta function and Igusa's zeta function. Indeed, the results for the topological zeta function imply the results for those 'finer' zeta functions.

\section{REFERENCES}

[Al] V. A. Alexeev, Boundedness and $K^{2}$ for log surfaces, International J. Math. 5 (1994), 779-810. MR 1298994 (95k:14048)

[DL1] J. Denef and F. Loeser, Caractéristique d'Euler-Poincaré, fonctions zêta locales et modifications analytiques, J. Amer. Math. Soc. 5 no. 4 (1992), 705-720. MR1151541 (93g:11118)

[DL2] J. Denef and F. Loeser, Motivic Igusa zeta functions, J. Alg. Geom. 7 (1998), 505-537. MR 1618144 (99j:14021)

[DL3] J. Denef and F. Loeser, Motivic exponential integrals and a motivic Thom-Sebastiani Theorem, Duke Mathematical Journal 99 (1999), 285-309. MR:1708026 (2000k:14006)

[De] J. Denef, Report on Igusa's local zeta function, Sém. Bourbaki 741, Astérisque 201/202/203 (1991), 359-386. MR1157848 (93g:11119)

[HL] K. Hoornaert and D. Loots, A computer program written in Maple to calculate Igusa's p-adic zeta function and the topological zeta funtion for non-degenerated polynomials, available on http://www.wis.kuleuven.be/algebra/kathleen.htm (2002).

[Ko1] J. Kollár, Log surfaces of general type; some conjectures, Classification of Algebraic Varieties, Contemp. Math. 162 (1994), 261-275. MR1272703 (95c:14042)

[Ko2] J. Kollár, Singularities of pairs, Summer Research Institute on Algebraic Geometry (Santa Cruz 1995), Amer. Math. Soc., Proc. Symp. Pure Math. 62.1 (1997), 221-287. MR 1492525 (99m:14033)

[Ku1] T. Kuwata, On log canonical thresholds of reducible plane curves, Amer. J. Math. 121 (1999), 701-721. MR1704476 (2001g:14047)

[Ku2] T. Kuwata, On log canonical thresholds of surfaces in $\mathbb{C}^{3}$, Tokyo J. Math. 22 (1999), 245-251. MR.1692033 (2000e:14055)

$\left[\mathrm{M}^{\mathrm{c}} \mathrm{KP}\right]$ J. $\mathrm{M}^{\mathrm{c}}$ Kernan and Yu. Prokhorov, Threefold thresholds, Manuscripta Math. 114 (2004), no. 3, 281-304. MR2075967 (2005g:14036)

[Pr1] Yu. Prokhorov, On log canonical thresholds, Comm. Algebra 29 (2001), 3961-3970. MR.1857023 (2002j:14019)

[Pr2] Yu. Prokhorov, On log canonical thresholds, II, Comm. Algebra 30 (2002), 5809-5823. MR.1941925 (2004c:14025)

[Se1] D. Segers, Smallest poles of Igusa's and topological zeta functions and solutions of polynomial congruences, K.U. Leuven Ph.D. thesis, available on http://www.wis.kuleuven.be/algebra/segers/segers.htm (2004).

[Se2] D. Segers, Lower bound for the poles of Igusa's p-adic zeta functions, Math. Annalen (to appear).

[Sh] V. Shokurov, 3-fold log flips, Izv. Russ. A. N. Ser. Mat. 56 (1992), 105-203. MR1162635 (93j:14012)

[SV] D. Segers and W. Veys, On the smallest poles of topological zeta functions, Compositio Math. 140 (2004), 130-144. MR2004126 (2004i:14004)

[Ve1] W. Veys, Poles of Igusa's local zeta function and monodromy, Bull. Soc. Math. France 121 (1993), 545-598. MR:1254752 (95b:11110)

[Ve2] W. Veys, Determination of the poles of the topological zeta function for curves, Manuscripta Math. 87 (1995), 435-448. MR.1344599 (97a:11192)

Departement Wiskunde, Celestijnenlaan 200B, B-3001 Leuven, Belgium

E-mail address: ann.lemahieu@wis.kuleuven.be

Departement Wiskunde, Celestijnenlaan 200B, B-3001 Leuven, Belgium

E-mail address: dirk.segers@wis.kuleuven.be

Departement Wiskunde, Celestijnenlaan 200B, B-3001 Leuven, Belgium

E-mail address: wim.veys@wis.kuleuven.be 